$\begin{array}{ll}\text { Mathematical Analysis } & \text { Vol. } 1(2020), \text { No. } 1,1-5 \\ \text { \& Convex Optimization } & \text { https: \ maco.lu.ac.ir } \\ & \text { DOI: } 10.29252 / \text { maco.1.1.1 }\end{array}$

Research Paper

\title{
ON THE EXISTENCE OF SOLUTIONS OF A GENERALIZED MONOTONE EQUILIBRIUM PROBLEM
}

\author{
HADI KHATIBZADEH* AND MAHNAZ REZAEI
}

\begin{abstract}
Blum and Oettli in their seminal paper studied the existence of equilibrium points for monotone bifunctions. In this work, we extend their main result by replacing monotone bifunction with a more general bifunction and prove the existence of an equilibrium point.
\end{abstract}

MSC(2010): 47H05; 47H04; 49J40; 49J53.

Keywords: Equilibrium problem, monotone bifunction, generalized monotonicity.

\section{Introduction and Background}

Throughout the paper, we assume that $X$ is a real Banach space with norm $\|\cdot\|$ and $K$ is a closed convex subset of $X$. By a bifunction we mean any function $f: K \times K \rightarrow$ $\mathbb{R}$ such that $f(x, x)=0, \forall x \in K$.

Definition 1.1. Let $f: K \times K \rightarrow \mathbb{R}$ be a bifunction. Consider the equilibrium problem (EP) of finding $\bar{x} \in K$ such that

$$
f(\bar{x}, y) \geq 0, \quad \forall y \in K
$$

$\bar{x}$ is called an equilibrium point for $f$ and $K$. The set of all equilibrium points for $f$ and $K$ is denoted by $E P(f, K)$.

Definition 1.2. Given a nonempty subset $\mathrm{K}$ of a Banach space $\mathrm{X}$, the bifunction $f: K \times K \rightarrow$ $\mathbb{R}$ is said to be

- monotone iff

$$
f(x, y)+f(y, x) \leq 0, \quad \forall x, y \in K .
$$

- pseudo-monotone if $f(x, y) \geq 0$ with $x, y \in K$, then $f(y, x) \leq 0$.

- quasi-monotone if $f(x, y)>0$ with $x, y \in K$, then $f(y, x) \leq 0$.

- $\theta$-monotone if there is a function $\theta: K \times K \rightarrow \mathbb{R}$ such that

$$
f(x, y)+f(y, x) \leq \theta(x, y)\|x-y\|, \quad \forall x, y \in K
$$

Date: Received: October 2, 2019, Accepted: March 13, 2020.

${ }^{*}$ Corresponding author. 
Example. Let $f:[0,+\infty) \times[0,+\infty) \rightarrow \mathbb{R}$ with $f(x, y)=x^{2}-x y$. Obviously it is not monotone but it is $\theta$-monotone with $\theta(x, y)=|x-y|$.

Existence of an equilibrium point for a monotone bifunction first studied by Blum and Oettli in [6]. An equilibrium point for a monotone bifunction can be a fixed point for a nonexpansive mapping, a solution of a variational inequality for a maximal monotone operator and a minimum point of a convex function. It has also some other interpratations in nonlinear problems. Therefore equilibrium problems unify several problems in nonlinear analysis and optimization (see [6]). Equilibrium problems for monotone and some variants of generalized monotone bifunctions has been studied by several authors (see for example [3, 4, 5, 7, 8, 9, 10, 11, 12, 13]). But the researchers have paid more attention to some generalized monotonicity of pseudo- and quasi-monotone type so far. Recently some general monotonicity conditions of different types for operators and bifunctions studied by authors (see [1, 2, 10, 11]). One of this conditions is $\theta$-monotonicity that was defined in above. In this paper, we extend the existence theorem of Blum and Oettli [6] form monotone bifunctions to $\theta$-monotone bifunctions.

\section{Main Results}

In this section we prove a basic existence result for the equilibrium problem in the case where $f(x, y)=g(x, y)+h(x, y)$. All assumptions on $g$ and $h$ are the same as assumed by Blum and Oettli [6] except monotonicity of $g$ that we replace it by $\theta$-monotonicity. Before the main theorem we recall a definition.

Definition 2.1. Let $\mathrm{K}$ and $\mathrm{C}$ be convex sets with $C \subset K$. Then $\operatorname{core}_{K} C$ is defined through $a \in \operatorname{core}_{K} C$ if $a \in C$, and $C \cap(a, y) \neq \varnothing$, for all $y \in K \backslash C$, where $(a, y)=$ $\{t a+(1-t) y ; 0<t<1\}$.

Theorem 2.2. Let the following assumptions hold

- $g: K \times K \rightarrow \mathbb{R}$ has the following properties:

$-g(x, x)=0, \quad \forall x \in K$

- For all $x, y \in K$ the function $t \in[0,1] \mapsto g(t y+(1-t) x, y)$ is upper-semicontinuous at $t=0 ;$ ( $g$ is called uper-hemicontinuous respect to the first argument);

$-g$ is convex and lower semicontinuous in the second argument;

$-g(x, y)+g(y, x) \leq \theta(x, y)\|x-y\|, \quad \forall x, y \in K,(\theta-$ monotonicity $)$ where

- $\theta: K \times K \rightarrow \mathbb{R}$ satisfies the following conditions:

$-\theta(x, x)=0, \forall x \in K$

- $\theta$ is upper semicontinuous respect to the second argument;

- $h: K \times K \rightarrow \mathbb{R}$ has the following properties:

$-h(x, x)=0, \quad \forall x \in K$

- $h$ is upper semicontinuous in the first argument;

$-h$ is convex in the second argument.

- (Coercivity condition) There exists $C \subset K$ nonempty, compact and convex such that for every $x \in C \backslash$ core $_{K} C$ there exists $a \in$ core $_{K} C$ such that $g(x, a)+h(x, a) \leq 0$.

Then there exists $\bar{x} \in C$ such that $0 \leq g(\bar{x}, y)+h(\bar{x}, y), \quad \forall y \in K$.

The proof goes over the following three lemmas, for which the hypotheses remain the same as for Theorem 2.2. 
Lemma 2.3. There exists $\bar{x} \in C$ such that

$$
g(y, \bar{x}) \leq \theta(y, \bar{x})\|y-\bar{x}\|+h(\bar{x}, y), \forall y \in C .
$$

Proof. For each $y \in C$ define

$$
S(y)=\{x \in C: g(y, x) \leq \theta(y, x)\|y-x\|+h(x, y)\}, \quad \forall y \in C
$$

By the assumptions on $g$ and $\theta, S(y)$ is closed and since $C$ is compact then $S(y)$ is compact for every $y \in C$. It is enough we show $\{S(y): y \in C\}$ has finite intersection property. Let $\left\{y_{i}: \quad i \in I\right\}$ be a finite and arbitrary subset of $C$ and $\zeta \in \operatorname{co}\left\{y_{i}: \quad i \in I\right\}$ (convex hull of $\left.\left\{y_{i}: \quad i \in I\right\}\right)$ be arbitrary. Therefore there are nonnegative scalers $\mu_{i}$ such that $\sum_{i \in I} \mu_{i}=1$ and $\zeta=\sum_{i \in I} \mu_{i} y_{i}$. Now suppose that $\zeta$ is not in $S\left(y_{i}\right), \forall i \in I$. i.e.

$$
g\left(y_{i}, \zeta\right)>\theta\left(y_{i}, \zeta\right)\left\|y_{i}-\zeta\right\|+h\left(\zeta, y_{i}\right)
$$

Multiplying both sides by $\sum_{i \in I} \mu_{i}$, then by the conditions on $h$, we get

$$
\begin{aligned}
\sum_{i \in I} \mu_{i} g\left(y_{i}, \zeta\right) & >\sum_{i \in I} \mu_{i}\left(\theta\left(y_{i}, \zeta\right)\left\|y_{i}-\zeta\right\|+h\left(\zeta, y_{i}\right)\right) \\
& \geq \sum_{i \in I} \mu_{i} \theta\left(y_{i}, \zeta\right)\left\|y_{i}-\zeta\right\|+\sum_{i \in I} \mu_{i} h\left(\zeta, y_{i}\right) \\
& \geq \sum_{i \in I} \mu_{i} \theta\left(y_{i}, \zeta\right)\left\|y_{i}-\zeta\right\|+h(\zeta, \zeta) \\
& \geq \sum_{i \in I} \mu_{i} \theta\left(y_{i}, \zeta\right)\left\|y_{i}-\zeta\right\|
\end{aligned}
$$

Adding both sides of the recent inequality by $\sum_{i \in I} \mu_{i} g\left(\zeta, y_{i}\right)$, and using the $\theta$-monotonicity of $g$, we get

$$
\begin{aligned}
\sum_{i \in I} \mu_{i} \theta\left(y_{i}, \zeta\right)\left\|y_{i}-\zeta\right\| & \geq \sum_{i \in I} \mu_{i}\left(g\left(y_{i}, \zeta\right)+g\left(\zeta, y_{i}\right)\right) \\
& >\sum_{i \in I} \mu_{i} \theta\left(y_{i}, \zeta\right)\left\|y_{i}-\zeta\right\|+\sum_{i \in I} \mu_{i} g\left(\zeta, y_{i}\right) \\
& \geq \sum_{i \in I} \mu_{i} \theta\left(y_{i}, \zeta\right)\left\|y_{i}-\zeta\right\|+g(\zeta, \zeta) \\
& =\sum_{i \in I} \mu_{i} \theta\left(y_{i}, \zeta\right)\left\|y_{i}-\zeta\right\|,
\end{aligned}
$$

which is a contradiction. Then there is $i \in I$ such that

$$
g\left(y_{i}, \zeta\right) \leq \theta\left(y_{i}, \zeta\right)\left\|y_{i}-\zeta\right\|+h\left(\zeta, y_{i}\right)
$$

Therefore for some $i \in I, \zeta \in S\left(y_{i}\right)$. Since $\zeta$ is an arbitrary element of $\operatorname{co}\left\{y_{i}: i \in I\right\}$, we conclude that

$$
\operatorname{co}\left\{y_{i}: i \in I\right\} \subset \cup_{i \in I} S\left(y_{i}\right) .
$$

Then by the KKM theorem, we get $\cap_{y \in C} S(y) \neq \varnothing$.

Lemma 2.4. The following statements are equivalent

(a) $\exists \bar{x} \in C, g(y, \bar{x}) \leq \theta(\bar{x}, y)\|\bar{x}-y\|+h(\bar{x}, y), \forall y \in C$;

(b) $\exists \bar{x} \in C, 0 \leq g(\bar{x}, y)+h(\bar{x}, y), \forall y \in C$. 
Proof. $(b) \Rightarrow(a)$ : From $\theta$-monotonicity of $g$, we have

$$
g(\bar{x}, y)+g(y, \bar{x})+h(\bar{x}, y) \leq \theta(\bar{x}, y)\|\bar{x}-y\|+h(\bar{x}, y)
$$

From (b), we get

$$
g(y, \bar{x}) \leq \theta(\bar{x}, y)\|\bar{x}-y\|+h(\bar{x}, y)
$$

$(a) \Rightarrow(b)$ : Let $y \in C$ be arbitrary, and take $x_{t}=t y+(1-t) \bar{x}$ and $0<t \leq 1$. Since $C$ is convex, then $x_{t} \in C$. Take $y=x_{t}$ in $(a)$, then

$$
g\left(x_{t}, \bar{x}\right) \leq \theta\left(\bar{x}, x_{t}\right)\left\|x_{t}-\bar{x}\right\|+h\left(\bar{x}, x_{t}\right)
$$

and

$$
\begin{aligned}
0 & =g\left(x_{t}, x_{t}\right) \\
& \leq t g\left(x_{t}, y\right)+(1-t) g\left(x_{t}, \bar{x}\right) \\
& \leq t g\left(x_{t}, y\right)+(1-t)\left(\theta\left(\bar{x}, x_{t}\right)\left\|\bar{x}-x_{t}\right\|+h\left(\bar{x}, x_{t}\right)\right) \\
& \leq t g\left(x_{t}, y\right)+(1-t)\left(\theta\left(\bar{x}, x_{t}\right)\left\|\bar{x}-x_{t}\right\|+t h(\bar{x}, y)+(1-t) h(\bar{x}, \bar{x})\right) \\
& =t\left(g\left(x_{t}, y\right)+(1-t) h(\bar{x}, y)\right)+(1-t) \theta\left(\bar{x}, x_{t}\right)\left\|x_{t}-\bar{x}\right\| \\
& =t\left(g\left(x_{t}, y\right)+(1-t) h(\bar{x}, y)\right)+(1-t) t \theta\left(\bar{x}, x_{t}\right)\|y-\bar{x}\| .
\end{aligned}
$$

Dividing both sides by $t$ and letting $t \rightarrow 0$, by semicontinuous of $g$ and $\theta$, we get the result.

Lemma 2.5. [6] Assume that $\Psi: K \rightarrow \mathbb{R}$ is convex, $x_{0} \in$ core $_{K} C, \Psi\left(x_{0}\right) \leq 0$, and $\Psi(y) \geq$ $0, \forall y \in C$. Then $\Psi(y) \geq 0, \quad \forall y \in K$.

Now we present the proof of Theorem 2.2.

Proof. From Lemma 2.3, we obtain $\bar{x} \in C$ with

$$
g(y, \bar{x}) \leq \theta(y, \bar{x})\|y-\bar{x}\|+h(\bar{x}, y), \forall y \in C
$$

From Lemma 2.4 follows that

$$
0 \leq g(\bar{x}, y)+h(\bar{x}, y), \forall y \in C
$$

Set $\Psi(\cdot):=g(\bar{x}, \cdot)+h(\bar{x},$.$) , then \Psi(\cdot)$ is convex and $\Psi(y) \geq 0, \forall y \in C$. If $\bar{x} \in C$, then set $x_{0}:=\bar{x}$. If $\bar{x} \in C \backslash$ core $_{K} C$, then set $x_{0}:=a$, where $a$ is as in coercivity assumption for $x=\bar{x}$. In both cases $x_{0} \in \operatorname{core}_{K} C$, and $\Psi\left(x_{0}\right) \leq 0$. Hence it follows from Lemma 2.5 that $\Psi(y) \geq 0, \forall y \in K$, i.e., $g(\bar{x}, y)+h(\bar{x}, y) \geq 0, \quad \forall y \in K$.

Corollary 2.6. Let the following assumptions hold

- $g: K \times K \rightarrow \mathbb{R}$ has the following properties:

$-g(x, x)=0, \quad \forall x \in K$

- For all $x, y \in K$ the function $t \in[0,1] \mapsto g(t y+(1-t) x, y)$ is upper-semicontinuous at $t=0$ (hemicontinuity);

- $g$ is convex and lower semicontinuous in the second argument;

$-g(x, y)+g(y, x) \leq \theta(x, y)\|x-y\|, \quad \forall x, y \in K(\theta-$ monotonicity); where

- $\theta: K \times K \rightarrow \mathbb{R}^{+}$has the following properties:

$-\theta(x, x)=0, \quad \forall x \in K$;

- $\theta$ is upper semicontinuous respect to the second argument. 
- There exists $C \subset K$ nonempty, compact and convex such that for every $x \in C \backslash$ core $_{K} C$ there exists $a \in$ core $_{K} C$ such that

$g(x, a) \leq 0$.

Then there exists $\bar{x} \in C$ such that $0 \leq g(\bar{x}, y), \forall y \in K$.

\section{Acknowledgments}

The authors are grateful to the referee for his(her) valuable comments.

\section{REFERENCES}

[1] M. H. Alizadeh, N. Hadjisavvas, and M. Roohi, Local boundedness properties for generalized monotone operators, J. Convex Anal., 19:49-61, 2012.

[2] M. H. Alizadeh M. Roohi, Some results on pre-monotone operators, Bull Iranian Math Soc., 43:2085-2097, 2017.

[3] M. Bianchi and R. Pini, Coercivity conditions for equilibrium problems, J. Optim. Theory Appl., 124:7992, 2005.

[4] M. Bianchi and S. Schaible, Generalized monotone bifunctions and equilibrium problems, J. Optim. Theory Appl., 90:31-43, 1996.

[5] M. Bianchi and S. Schaible, Equilibrium problems under generalized convexity and generalized monotonicity, J. Global Optim., 30:121-134, 2004.

[6] E. Blum and W. Oettli, From optimization and variational inequalities to equilibrium problems, Math. Student, 63:2951-2955, 1994.

[7] O. Chadli, Z. Chbani, H. Riahi, Equilibrium problems with generalized monotone bifunctions and applications to variational inequalities, J. Optim. theory Appl., 105:299-323, 2000.

[8] O. Chadli, A. Lahmdani and J.C. Yao, Existence results for equilibrium problems with applications to evolutions equations, Appl. Anal., 94:1709-1735, 2015.

[9] J. Cotrina and Y. Garcia, Equilibrium Problems: Existence results and applications, Set-valued Var. Anal., 26:159-177, 2018.

[10] A. Iusem, G. Kassay and W. Sosa, On certain conditions for the existence of solutions of equilibrium problems, Math. Program. Ser B., 116:259-273, 2009.

[11] A. Iusem, G. Kassay and W. Sosa, An existence result for equilibrium problems with some surjectivity consequences. J. Convex Anal., 16:80-826, 2009.

[12] G. Kassay and M. Mihaela, Existence results for vector equilibrium problems given by a sum of two functions, J. Global Optim., 63:195-211, 2015.

[13] I. V. Konnov and D. A. Dyabilkin, Nonmonotone equilibrium problems: coercivity conditions and weak regularization, J. Glob. Optim., 49:575-587, 2011.

(Hadi Khatibzadeh) Department of Mathematics, University of Zanjan, Zanjan, Iran.

Email address: hkhatibzadeh@znu.ac.ir

(Mahnaz Rezaei) Department of Mathematics, University of Zanjan, Zanjan, Iran.

Email address: mahnaz.rezaei615@gmail.com 